\title{
Spektral abstimmbare IR-Sensoren für die industrielle Prozessmesstechnik und die medizinische Gasanalyse
}

Martin Ebermann, Norbert Neumann

InfraTec GmbH, Gostritzer Str. 61 - 63, 01217 Dresden

0351871 8625, m.ebermann@infratec.de

Zusammenfassung: Mikrospektrometer besitzen ein großes Potenzial in der IR-Absorptionsspektroskopie, insbesondere für die Mehrkomponentenanalyse. Im Beitrag werden durchstimmbare IR-Detektoren für Spektralbereiche im mittleren und langwelligen Infrarot vorgestellt, welche auf spektral breitbandigen pyroelektrischen Detektoren und mikromechanischen Fabry-Pérot-Filtern als wellenlängenselektives Element basieren. Eine spezielle Dual-Band-Konfiguration ist für die simultane Detektion in zwei Spektralbereichen geeignet. Durch Verbesserungen des elektromechanischen Designs ist es außerdem gelungen, die Beschleunigungsempfindlichkeit der Filter signifikant zu reduzieren. Es wird kurz auf den Aufbau eines Mikrospektrometers und möglicher Betriebsweisen der Detektoren eingegangen um anschließend einige ausgewählte Beispielapplikationen aus der medizinischen und industriellen Analytik vorzustellen. Abschließend wird ein Ausblick auf eine neue Generation höher auflösender und schnellerer FPSpektrometer gegeben.

\section{Einführung}

Das Verfahren der Infrarotspektroskopie als physikalisches Sensorprinzip hat seit langem einen festen Platz in der Sensorik erobert, um Stoffkonzentrationen und Zusammensetzungen sehr selektiv und langzeitstabil zu bestimmen. Ein Haupanwendungsgebiet ist die Gasanalyse, so z. B. in der Sicherheitstechnik für die Detektion giftiger oder explosiver Gase, in der Umwelttechnik zur Messung von Schadstoffemissionen oder der Zusammensetzung der Atmosphäre, in der Klima- und Gebäudetechnik zur Überwachung und Regelung der Luftqualität in Innenräumen, in der Energietechnik für die Analyse von Brenngasen bzw. zur Bestimmung des Energiegehalts sowie in der Medizintechnik zur Atemgasanalyse. Daneben wird die IR-Spektroskopie auch für Flüssigkeits- und Feststoffanalysen angewandt. Beispiele hierfür sind die Prozessüberwachung und -regelung in der Chemie- und Lebensmittelindustrie sowie die Identifikation von Kunststoffen oder von Filmen bzw. Beschichtungen auf Oberflächen. Konventionelle NDIR-Analysatoren bestehen meist aus einer breitbandigen InfrarotStrahlungsquelle, einer durchstrahlten Probenkammer und einem Detektor. Zur Analyse werden häufig ein Mess- und Referenzkanal, oft auch noch zusätzliche Kanäle verwendet. Ihre spektrale Empfindlichkeit wird mit Hilfe von Schmalbandfiltern vorgegeben, deren Bandbreite typischerweise einige Prozent der Wellenlänge beträgt. In der praktischen Umsetzung werden meist mehrkanalige Detektoren eingesetzt, aber auch Filterräder in Kombination mit einem Einzeldetektor finden noch vielfach Verwendung [1,2]. Mikromechanisch hergestellte und elektrisch durchstimmbare Fabry-Perot-Filter (FPF) bieten nun die Möglichkeit, die Erfassung kontinuierlicher Spektren mit dem einfachen und robusten Aufbau der NDIRAnalysatoren zu verbinden. Da die FPF direkt in die Detektorgehäuse eingebaut werden können, ergibt sich ein enormer Miniaturisierungsvorteil [3-6]. Weitere Vorteile sind die kostengünstige Fertigung mit den Methoden der Mikrosystemtechnik und die relativ einfache Adaption bestehender Gerätedesigns, um schnell und mit geringem Aufwand zu neuen messtechnischen Lösungen zu gelangen.

In der Gasanalyse wird fast ausnahmslos in den beiden atmosphärischen Fenstern von $3-5 \mu \mathrm{m}$ (MWIR) und 8-12 $\mu \mathrm{m}$ (LWIR) Wellenlänge gemessen, weil hier keine störenden Einflüsse durch die starken Banden des Wasserdampfes auftreten. Eine Messung im MWIR ist normalerweise gegenüber höheren Wellenlängen bevorzugt, da eine größere Auswahl an leistungsfähigen und preiswerten Komponenten auf Strahler- und Empfängerseite sowie bei den Fenstermaterialien besteht. Nachteilig im LWIR ist, dass dies der Bereich der thermischen Strahlung bei Umgebungstemperatur ist (thermisches Infrarot). Die Messungen sind deshalb viel empfindlicher in Bezug auf die Hintergrundstrahlung bzw. Änderungen der Umgebungstemperatur. Ein Großteil der technisch relevanten Gase besitzt Absorptionsbanden im MWIR, welche sich aber innerhalb bestimmter Stoffklassen oft sehr stark ähneln und deshalb schlecht zu unterscheiden sind. Die Messung ist demnach zwar sehr empfindlich aber nicht - oder nur bei sehr hoher spektraler Auflösung - auch selektiv. In höheren Wellenlängenbereichen (sogenannter „Fingerprint“Bereich) sind sie dagegen wesentlich spezifischer und damit selektiver messbar. Für die Identifikation der 
Komponenten in unbekannten Gasmischungen wird normalerweise eine sehr hohe Auflösung gefordert um kleinste Details im Absorptionsspektrum sichtbar zu machen. Für die genaue quantitative Messung bekannter Komponenten steht dagegen ein hoher Signal-Rausch-Abstand im Vordergrund. Dafür ist ein hoher optischer Durchsatz Voraussetzung, was zwangsläufig mit einer reduzierten Auflösung einhergeht. Viele Moleküle besitzen allerdings eher breite Absorptionsbanden ohne feinaufgelöste Linienstruktur. Noch deutlicher ist dieser Effekt bei der Analyse von Flüssigkeiten und Feststoffen. Für viele Anwendungen ist daher eine spektrale Auflösung von wenigen zehn Nanometern, wie sie mit mikromechanischen FP-Filtern erreicht werden kann, völlig ausreichend.

In den folgenden Abschnitten wird der Stand der kommerziell verfügbaren FP-Filter für verschiedene Wellenlängenbereiche im mittleren und langwelligen Infarot kurz zusammengefasst. Es werden Perspektiven für künftige Verbesserungen und neue Generationen von FP-Filtern aufgezeigt und einige exemplarische Anwendungen in verschiedenen Spektralbereichen vorgestellt.

\section{Aufbau und Funktionsweise mikromechanischer FP-Spektrometer}

\subsection{Grundgleichungen des Fabry-Pérot-Interferometers}

Das Fabry-Perot-Interferometer (FPI) besteht aus zwei ebenen Spiegelplatten (Reflektoren) welche in einem Abstand $d$ parallel zueinander angeordnet sind und ein Medium mit der Brechzahl $n$ einschließen. Die beiden Reflektoren sind jeweils gekennzeichnet durch ihren Transmissionsgrad $T$, den Reflexionsgrad $R$, Absorptionsgrad $A$ sowie eine Phasenverschiebung der Reflexion $\phi$. Die Reflektoreigenschaften $R, T, A$ und $\phi$ sind im Allgemeinen wellenlängenabhängig.

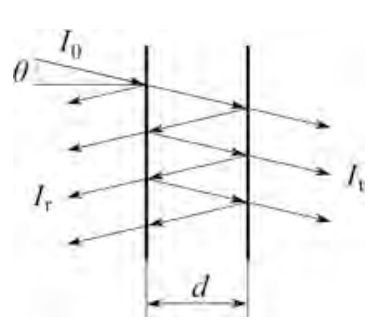

$$
\begin{aligned}
& T(\lambda)=\frac{I_{t}}{I_{0}}=\frac{T_{\max }}{1+\frac{4 R}{(1-R)^{2}} \sin ^{2}\left(2 \pi n d \frac{1}{\lambda} \cos \theta-\phi\right)} \\
& T_{\max }=\left(1-\frac{A}{(1-R)}\right)^{2}
\end{aligned}
$$

Bild 1: Prinzipaufbau und Grundgleichungen des Fabry-Pérot Interferometers

Das Bild 1 illustriert die Grundanordnung sowie die Definition der Größen und Parameter. Im Resonatorspalt entstehen Vielfachinterferenzen, wobei nur die Strahlung transmittiert wird, welche die Resonanzbedingungung erfüllt. Die Transmission $T(\lambda)$ des FPI wird durch die sogenannte Airy-Formel (GIn. 1, 2) beschrieben. Eine vollständige Darstellung der Theorie kann z. B. in [7] nachgelesen werden. Das FPI kann als spektral durchstimmbares schmalbandiges Filter benutzt werden (FP-Filter), indem die Resonanzwellenlänge durch Variation des Spaltes $d$ eingestellt wird. In Bild 2 sind berechnete Spektren ${ }^{1}$ für ein FP-Filter erster Ordnung $(m=1)$ mit Variation des Abstands im Bereich $3-5,5 \mu \mathrm{m}$ dargestellt. In dieser Konfiguration ist das der theoretisch maximale Abstimmbereich, begrenzt durch den freien Spektralbereich FSR (free spectral range) - den Abstand zur nächst höheren Interferenzordnung.

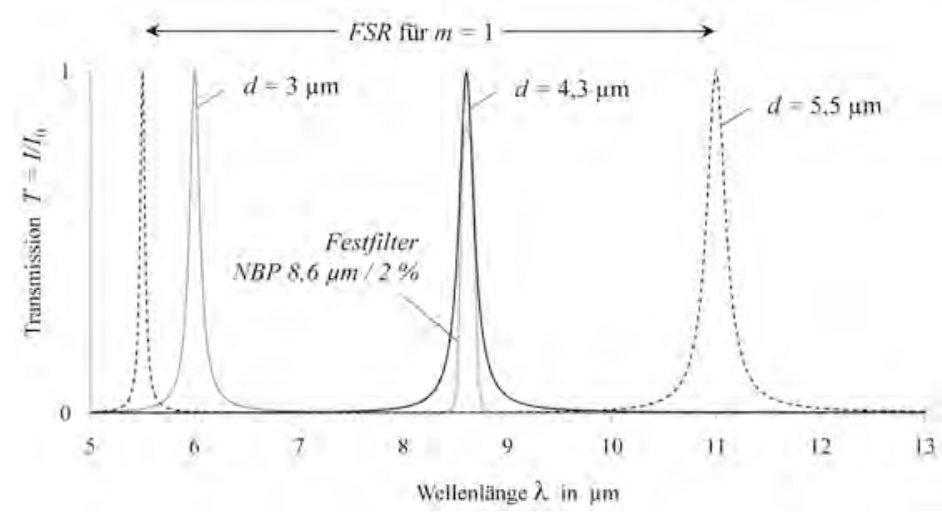

\footnotetext{
${ }^{1}$ Berechnung unter Annahme stark idealisierter Bedingungen
} 
Das spektrale Auflösungsvermögen entspricht der Filterbandbreite FWHM (full width at half maximum). Es hängt maßgeblich vom Reflexionsgrad der Spiegel sowie von Defekten wie Verkrümmung, Schiefstellung oder Rauheit der Reflektoren ab [7-9]. Zusätzlich zu den FP-Filterspektren zeigt Bild 2 das reale Transmisssionspektrum eines typischen kommerziellen Schmalbandfilters (NBP) mit der gleichen nominellen Bandbreite. Aus der Form der Spektren wird offensichtlich, dass das FP-Filter im Blockungsbereich, d. h. bei der Unterdrückung benachbarter Spektralbereiche, zunächst im Nachteil ist. Dies kann aber wettgemacht werden, wenn das FP-Filter konsequent zur Erfassung kontinuierlicher Spektren eingesetzt wird und nicht nur auf diskreten Spektrakanälen.

\subsection{FP-Spektrometermodul für den Spektralbereich $3-5 \mu \mathrm{m}$}

Bild 3a zeigt den schematischen Aufbau eines durchstimmbaren FP-Filters, dass aus mikromechanisch strukturierten Ober- und Unterteilen gefügt wurde. Zwei Bragg-Reflektoren auf dicken Trägern bilden den Luftspalt. Der obere Reflektorträger ist beweglich an Federn befestigt. Durch Anlegen einer Spannung zwischen dem oberen Reflektorträger und der unteren Kontrollelektrode wird der Luftspalt verringert und das Filter spektral durchgestimmt.

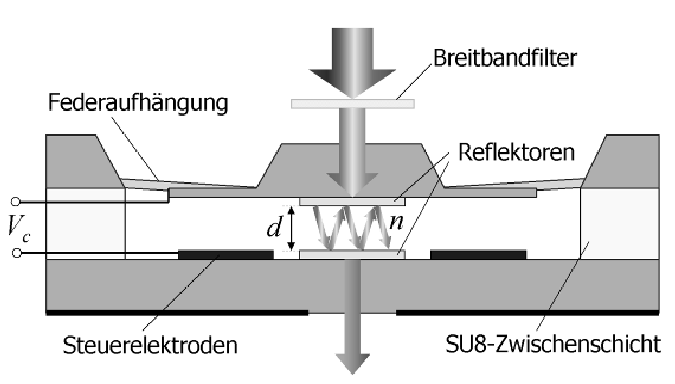

a)

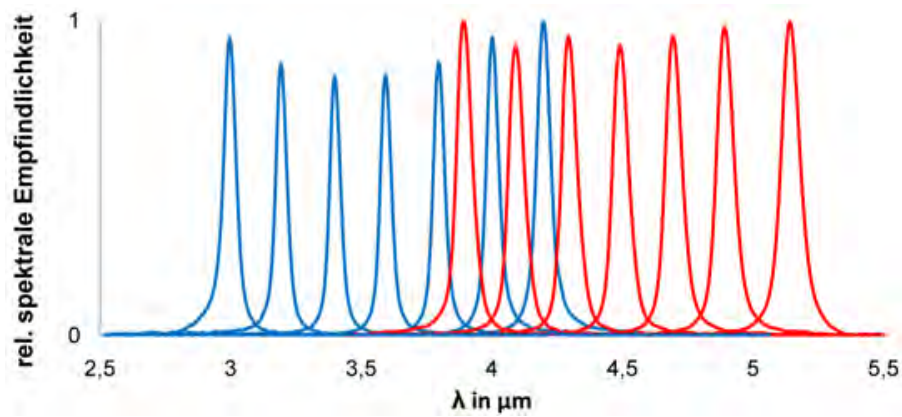

b)

Bild 3: a) Prinzipaufbau des mikromechanischen Fabry-Pérot-Interferometers, b) relative spektrale Empfindlichkeit der Detektortypen LFP-3041 L-337 (blau) und LFP-3950L-337 (rot) (FTIR-Spektrometer, $8 \mathrm{~cm}^{-1}, \pm 4^{\circ}$ )

Das Filter wird mit den Technologien der Volumenmikromechanik hergestellt [10] . Zunächst werden die Reflektorschichten hergestellt und anschließend strukturiert. Ober- und Unterteil werden schließlich mit Hilfe einer Zwischenschicht aus SU-8 gebondet. Die hohe Steifigkeit der dicken Reflektorträger hilft Deformationen gering zu halten. Dadurch kann trotz der großen Filterapertur von $\varnothing 1,9 \mathrm{~mm}$ eine Finesse im Bereich von $40 \ldots 60$ erreicht werden. Die Herstellung der Bragg-Reflektoren aus $\mathrm{SiO}_{2}$ und PolySilizium ist voll prozesskompatibel zur üblichen MEMS-Technologie. Zwei Filtertypen mit verschiedenen optischen Designs für die beiden Teilbereiche von 3-4,1 $\mu \mathrm{m}$ (short) und 3,9-5 $\mu \mathrm{m}$ (long) werden derzeit als serienreife Bauelemente angeboten. In Tabelle 1 sind die wichtigsten spektralen Parameter aufgelistet. Die spektrale Empfindlichkeit der beiden Detektortypen ist Bild 3b dargestellt.

Der integrierte pyroelektrische Detektor basiert auf bewährter $\mathrm{LiTaO}_{3}$-Technologie. Der Detektor besitzt ein $2 \times 2 \mathrm{~mm}^{2}$ großes Sensorelement, ist parallelkompensiert und arbeitet im Strombetrieb (Transimpedanz-Verstärker). Der Detektor weist eine große spezifische Detektivität von ca. $4 \cdot 10^{8} \mathrm{~cm}$ $\mathrm{Hz}^{1 / 2} / \mathrm{W}$ bei einer Modulationsfrequenz von $10 \mathrm{~Hz}$ auf. Die minimierte Rückkoppelkapazität sorgt für eine hohe Empfindlichkeit von über $100 \mathrm{kV} / \mathrm{W}$ und einen flachen Amplitudengang bis zu einigen zehn Hertz.

Tabelle 1: Spektrale Filtereigenschaften (FTIR Spektrometer, $8 \mathrm{~cm}^{-1}, \pm 4$ ), rechts: Detektormodul im TO8-Gehäuse

\begin{tabular}{lcc} 
& LFP-3041L-337 (short) & LFP-3950L-337 (long) \\
\hline Spektralbereich & $3-4,1 \mu \mathrm{m}$ & $3,9-5 \mu \mathrm{m}$ \\
Halbwertsbreite & $80 \pm 20 \mathrm{~nm}$ & $100 \pm 20 \mathrm{~nm}$ \\
Peak-Transmission & $>50 \%$ \\
Out-of-band Blockung & $<0,5 \%$ \\
Effektive Finesse & $40 \ldots 60$ \\
\hline
\end{tabular}

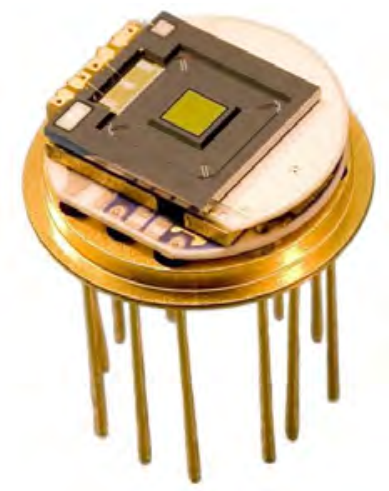




\subsection{Schnelles kontinuierliches Abstimmen im Sweep-Modus}

Der sogenannte Step-Scan-Modus entspricht im Grundprinzip dem herkömmlichen Messverfahren. Es wird eine gleichförmig modulierte Strahlungsquelle verwendet, die Auswertung des Detektorsignals erfolgt mit Hilfe der Lock-in- bzw. FFT-Technik. Zusätzlich dazu wird nun zwischen den einzelnen Messpunkten die Filterwellenlänge sequentiell eingestellt, bis das vollständige Spektrum erfasst ist. Für die Aufnahme eines Spektrums werden einige Sekunden benötigt.

Beim Sweep-Modus wird das Filter kontinuierlich durchgestimmt. Diese Betriebsart hat den Vorteil, Messungen deutlich zu beschleunigen, da das Filter innerhalb kurzer Zeit $(<1 \mathrm{~s})$ komplett durchgestimmt werden kann. Ein weiterer großer Vorteil ist, dass bei Bedarf wesentlich leistungsstärkere Strahler eingesetzt werden können, da die Notwendigkeit der direkten Modulation entfällt. Unter Umständen kann eine „heiße“ Probe sogar direkt gemessen werden.

Pyroelektrische Detektoren sind aufgrund ihrer hohen Zeitkonstanten und der frequenzabhängigen Empfindlichkeit nur bedingt für den Sweep-Modus geeignet. Höhere Bandbreite und eine frequenzunabhängige Empfindlichkeit bieten demgegenüber photoleitende und photovoltaische IRDetektoren, wie z.B. PbSe-Widerstände und InAs-Dioden. Erste Prototypen abstimmbarer Sensoren mit integriertem PbSe-Fotowiderstand sind aufgebaut und für eine schnelle $\mathrm{CO}_{2}$-Messung im Sweep-Modus getestet worden. Dies kann z. B. für atemzugsaufgelöste Messungen beim Patientenmonitoring in der medizinischen Gasanalyse genutzt werden. Bild 4 zeigt das Messprinzip und die bei der Messung aufgenommenen Zeitsignale des Detektors.

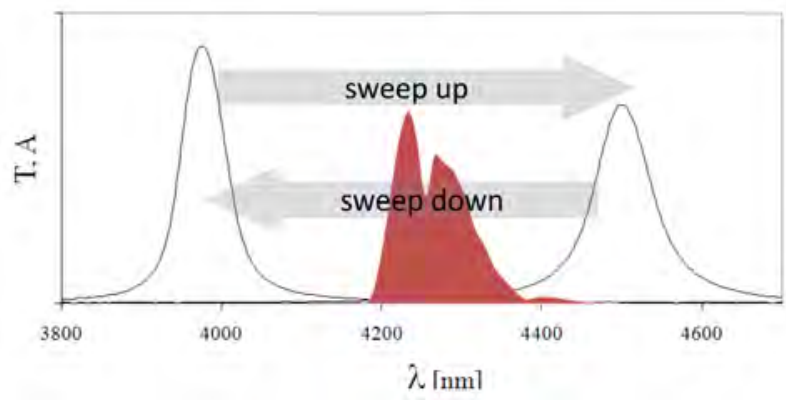

a)

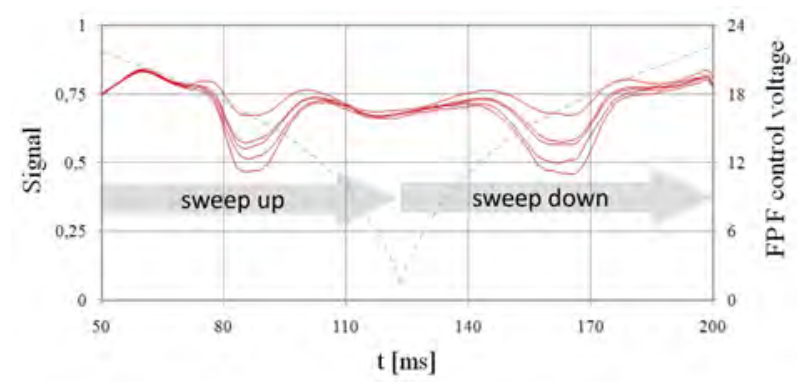

b)

Bild 4: a) Prinzip der schnellen $\mathrm{CO}_{2}$-Messung im Sweep-Modus, Filterspektren und Absorptionsspektrum des $\mathrm{CO}_{2}$ (rot), b) direkte Gewinnung des Absorptionsspektrums aus dem Zeitsignal

Das FP-Filter wird kontinuierlich und näherungsweise linear über die Absorptionsbande des CO2 durchgestimmt. Das Detektorsignal wird in beiden Scanrichtungen („up“ und „down“) ausgewertet. Da der Detektor quasi DC betrieben werden kann und einen flachen Frequenzgang besitzt, repräsentiert das Zeitsignal direkt das gesuchte Transmissionsspektrum. Ein kompletter Zyklus dauert hier $200 \mathrm{~ms}$, d. h. es ergibt sich eine effektive Messfrequenz für das komplette Spektrum von $10 \mathrm{~Hz}$. Gegenüber dem konventionellen Step-Scan-Modus ist dies bereits eine erhebliche Beschleunigung. Die Geschwindigkeit bei der hier gezeigten Messung ist derzeit noch durch die Dynamik des FP-Filters begrenzt. Dies soll zukünftig noch verbessert werden, so dass eine weitere Beschleunigung um ca. den Faktor $5 \ldots 10$ möglich wird.

\subsection{FP-Filter für Spektralbereiche bis $\mathbf{1 1} \mu \mathrm{m}$ und Dual-Band-Fähigkeit}

Die Notwendigkeit, in Wellenlängenbereichen $>5 \mu \mathrm{m}$ messen zu können, ist bereits in Abschnitt 1 erläutert worden. Auf der Basis einer neuen Reflektortechnologie wurden in den letzten Jahren entsprechende FP-Filter entwickelt [11]. Der komplette Spektralbereich von 3-11 $\mu \mathrm{m}$ lässt sich nach dem Stand der Technik allerdings nicht innerhalb einer einzigen Interferenzordnung des FP-Filters abdecken.

Eine der Neuentwicklungen zielt auf den Anwendungsbereich der Flüssigkeitsanalyse im Spektralbereich $5,5-8 \mu \mathrm{m}$ ab. FP-Filter und komplettes Detektormodul entsprechen im Aufbau den im vorigen Abschnitt vorgestellten Typen für den Bereich 3-5 $\mu \mathrm{m}$. Typische Filterspektren sind in Bild 5 dargestellt. Das Filter wird in der ersten Interferenzordnung mit einer Steuerspannung von ca. $50 \mathrm{~V}$ durchgestimmt. Es wird eine spektrale Auflösung von unter $100 \mathrm{~nm}$ erreicht. 


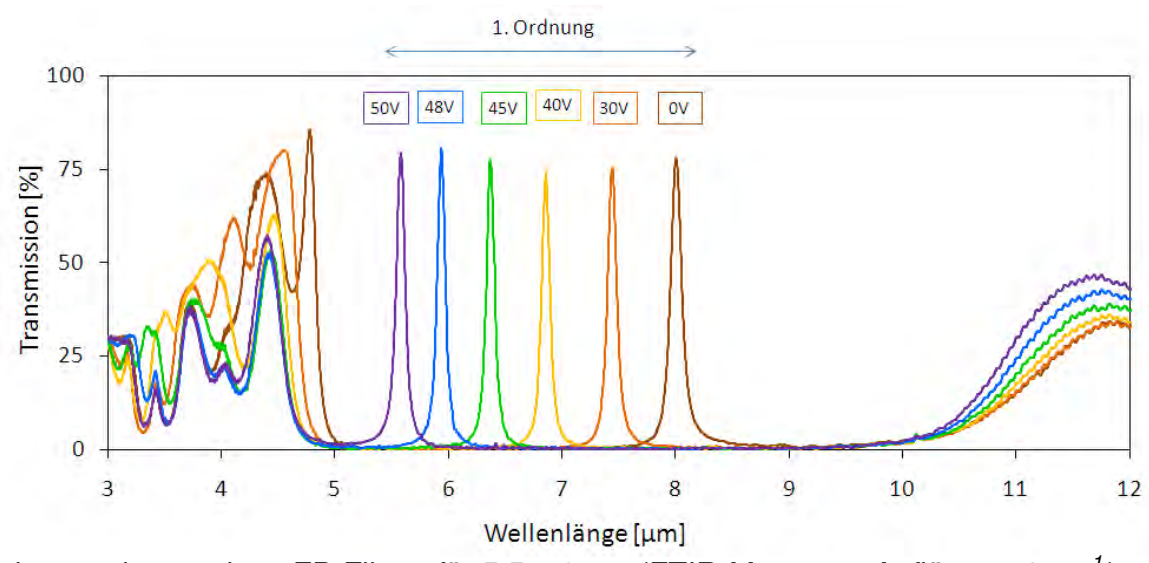

Bild 5: Transmissionsspektrum eines FP-Filters für 5,5-8 $\mu$ m (FTIR-Messung, Auflösung $4 \mathrm{~cm}^{-1}$ )

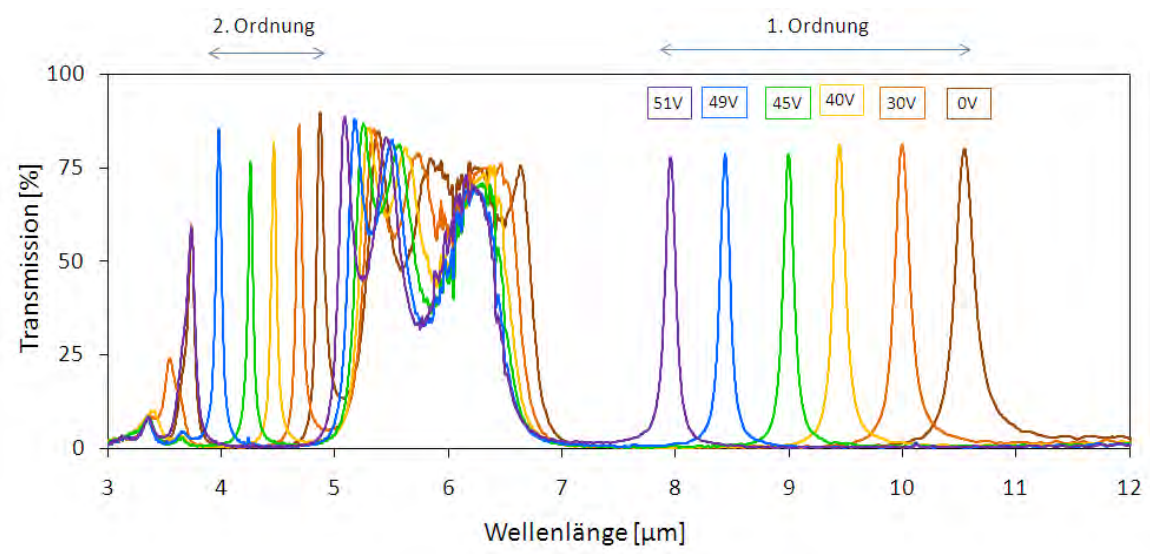

Bild 6: Transmissionsspektrum eines DB-FP-Filters (FTIR-Messung, Auflösung $4 \mathrm{~cm}^{-1}$ )

Für Messaufgaben, bei denen Banden im MWIR und LWIR gleichzeitig erfasst werden sollen, wurde der neue Ansatz eines Dual-Band Filters entwickelt. Die Grundidee beruht darauf, mittels eines speziell entworfenen Dual-Band-Reflektors zwei Interferenzordnungen simultan zu nutzen. Das Prinzip wurde erstmalig an Mustern demonstriert, welche speziell für die Messung von Anästhesiegasen in den Spektralbereichen 3,9-4,8 $\mu \mathrm{m}$ und $8-10,5 \mu \mathrm{m}$ entworfen wurden. In Bild 6 sind typische Spektren eines solchen Dual-Band-Filters dargestellt. Es konnten sehr hohe Transmissionswerte von bis zu $80 \%$ in beiden Bändern, und niedrige Halbwertsbreiten von FWHM $\leq 120 \mathrm{~nm}$ im oberen und FWHM $\leq 60 \mathrm{~nm}$ im unteren Spektralbereich erreicht werden.

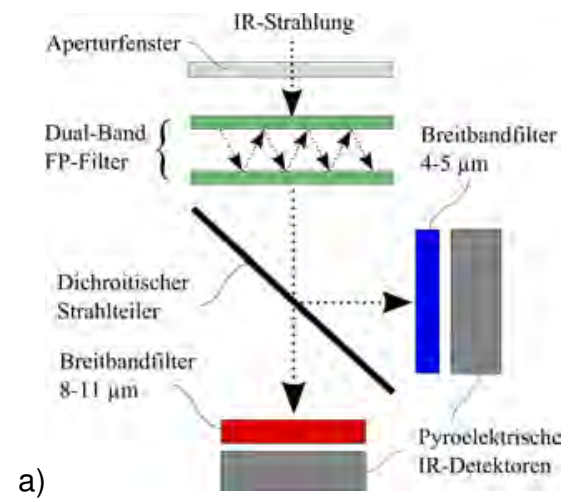

b)

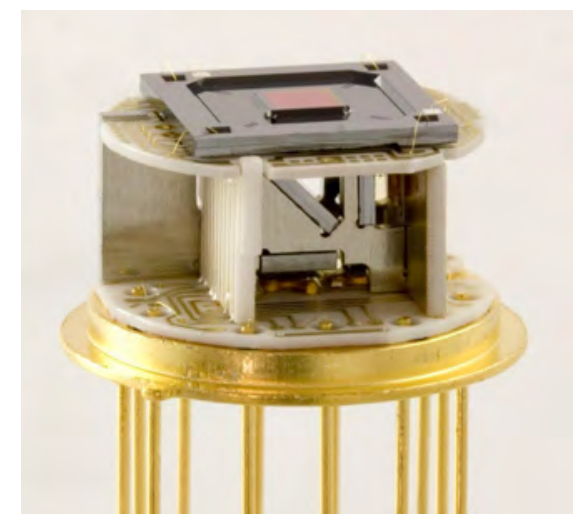

Bild 7: a) Prinzipaufbau des DB-Spektrometermoduls, b) Prototyp im TO8-Gehäuse

Die Trennung der beiden Interferenzordnungen wird innerhalb des DB-Detektormoduls durch einen dichroitischen Strahlteiler realisiert, der im Winkel von $45^{\circ}$ angeordnet ist. Im Gegensatz zu einem 50/50Strahlteiler bleiben die Signalverluste so in einem Rahmen von 5-10\%. Zusätzliche Breitbandfilter vor den Detektoren werden benötigt, um unerwünschte Strahlungsanteile zu blocken. Der prinzipielle Aufbau des DB-Detektors ist in Bild 7a dargestellt, Bild 7b zeigt einen Prototypen des kompletten DBDetektormoduls. 
Tabelle 3: Spektrale Filtereigenschaften der neuen Single-Band und Dual-Band-Filter, derzeitiger Entwicklungsstand

\begin{tabular}{lccc}
\hline & $\begin{array}{c}\text { DB-Filter } \\
\text { 2. Ordnung }\end{array}$ & $\begin{array}{c}\text { SB-Filter } \\
\text { 1. Ordnung }\end{array}$ & $\begin{array}{c}\text { DB-Filter } \\
\text { 1. Ordnung }\end{array}$ \\
\hline Abstimmbereich $[\mu \mathrm{m}]$ & $3,9-4,8$ & $5,5-8$ & $8-10,5$ \\
Ansteuerspannung [V] & & $\approx 50 \mathrm{~V}$ & \\
Halbwertsbreite [nm] & $50 \ldots 80$ & $80 \ldots 120$ & $105 \ldots 230$ \\
Peak-Transmission [\%] & $65 \ldots 90$ & $70 \ldots 80$ & $75 \ldots 85$ \\
Reflexionsfinesse & $\leq 35$ & $\leq 75$ & $\leq 70$ \\
Effektive Finesse & $\leq 23$ & $\leq 60$ & $\leq 56$ \\
\hline
\end{tabular}

\subsection{Reduzierung der Beschleunigungsempfindlichkeit}

Die dielektrischen Reflektoren werden auf $300 \mu \mathrm{m}$ dicken Si-Substraten aufgebracht, die die Verwölbung durch Schichtstress begrenzen, deren Masse aber nicht vernachlässigt werden kann, wenn das Filter statischen und dynamischen Beschleunigungen ausgesetzt wird. Signifikante Verbesserungen hin zu reduzierter Beschleunigungsempfindlichkeit und gleichzeitig reduzierter Ansteuerspannung kann man erreichen, wenn man zwei an Federn befestigte Reflektorträger benutzt [12, 13]. Bild 8a zeigt den schematischen Querschnitt des derart modifizierten Aufbaus.

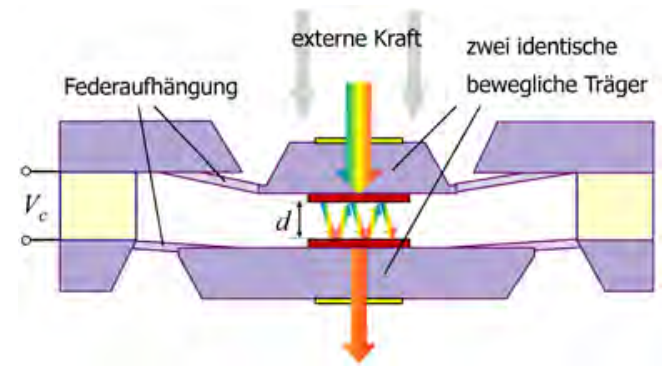

a)

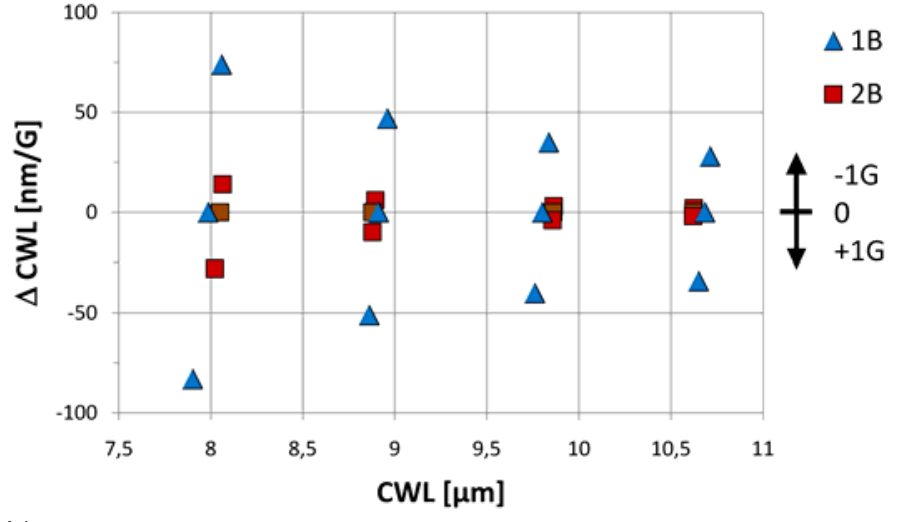

b)

Bild 8: a) Schematischer Querschnitt eines FP-Filters mit zwei an Federn befestigten Reflektorträgern, b) Vergleich des Schwerefeldeinflusses auf die Zentralwellenlänge von FP-Filtern mit einem (1B, blau) bzw. zwei beweglichen (2B, rot) Reflektorträgern braune Quadrate?

Das Prinzip der Nutzung von zwei beweglichen Reflektorträgern (2B) wurde erstmalig an Dual-BandFiltern getestet. Das optische Grunddesign (Reflektoren, Abstimmbereich) entspricht der oben gezeigten Variante mit einem beweglichen Reflektorträger (1B). Die Wirkung der Erdbeschleunigung auf die Zentralwellenlänge der beiden unterschiedlichen Filterdesigns ist in Bild $8 \mathrm{~b}$ für den vollständigen Abstimmbereich der ersten Ordnung dargestellt. Für das 1B-Filter müssen dazu bis zu $50 \mathrm{~V}$, beim 2BFilter lediglich $40 \mathrm{~V}$ Steuerspannung angelegt werden. Die Lageempfindlichkeit konnte hier um den Faktor 3-4 verbessert werden. Dies gilt nicht nur für den statischen Fall, d. h. Lageänderungen im Schwerefeld, sondern ebenso für niederfrequente Vibrationen (wenige $10 \mathrm{~Hz}$ ). Höherfrequente Beschleunigungen werden dagegen sehr wirksam durch die engen Spalte zwischen den Trägern, sowie zwischen Trägern und Rahmen bedämpft.

Dass das 2B-Filter noch nicht vollständig unempfindlich gegen Beschleunigungen ist, liegt einerseits an Toleranzen von Steifigkeiten und Massen der beiden Träger. Außerdem besteht noch eine gewisse Asymmetrie im Design, da die elektrostatische Kraft zur Ansteuerung auch zwischen Trägern und Rahmen wirkt. In nächster Zukunft werden hier noch einmal deutliche Verbesserungen erwartet. 


\section{Anwendung von FP-Spektrometern in der IR-Spektroskopie}

\subsection{Messung von Anästhesiegasen mit einem DB-FP-Spektrometer}

In der modernen Anästhesie werden Flurane (Enfluran, Desfluran, Sevofluran, Halothan, Isofluran) und Lachgas $\left(\mathrm{N}_{2} \mathrm{O}\right)$ eingesetzt. Die Flurane zeigen relative schwache Absorptionen im MWIR, im LWIR sind sie dagegen sehr gut messbar. Die spektralen Details in der Struktur der Spektren liegen in der Größenordnung von $100 \ldots 200 \mathrm{~nm}$. Daraus ergibt sich die Forderung nach einer entsprechenden spektralen Auflösung für eine selektive Messung. Als wichtiger Parameter für den Zustand des Patienten wird während der Narkose auch das $\mathrm{CO}_{2}$ in der Atemluft überwacht. Eine intensive Absorptionsbande des $\mathrm{CO}_{2}$ liegt bei einer Wellenlänge von ca. 4,25 $\mu \mathrm{m}$, also im MWIR. Der Bereich $8-12 \mu \mathrm{m}$ ist dagegen frei von Absorptionen des $\mathrm{CO}_{2}$. $\mathrm{N}_{2} \mathrm{O}$ kann sehr gut sowohl im MWIR bei ca. 4,66 $\mu \mathrm{m}$ als auch im LWIR knapp unterhalb von $8 \mu \mathrm{m}$ Wellenlänge gemessen werden, interferiert aber bei letzterer mit den Absorptionsbanden der Flurane und des Wasserdampfes. Zur Verdeutlichung sind in Bild 9 Transmissionsspektren der genannten Gase dargestellt. Gleichzeitig ist auch der in der Atemluft des

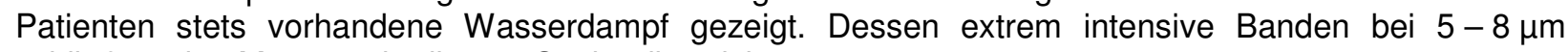
schließen eine Messung in diesem Spektralbereich aus.

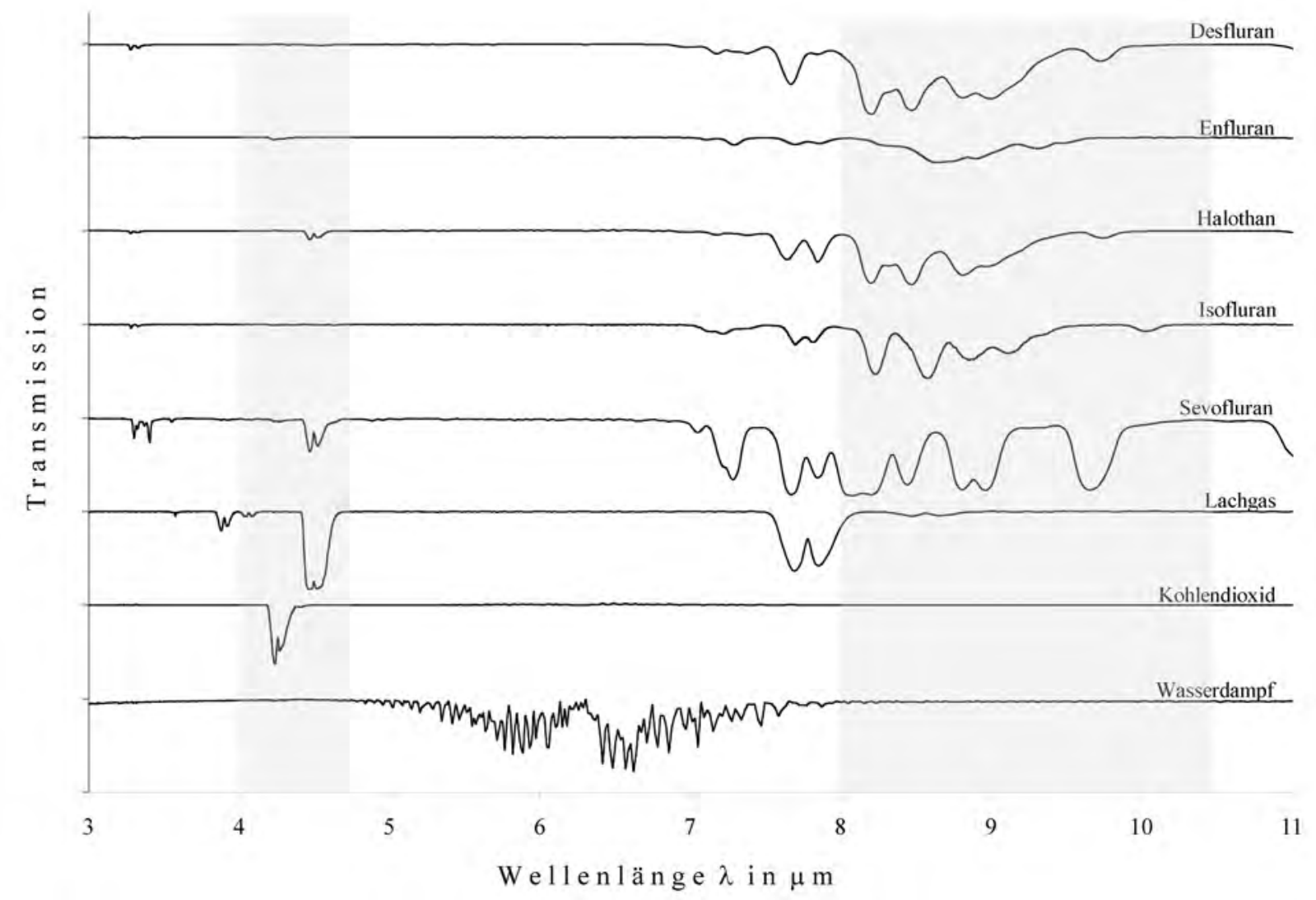

Bild 9: Transmissionsspektren der Anästhesiegase, Kohlendioxid und Wasserdampf (Messung mit FTIR-

Spektrometer, Auflösung $4 \mathrm{~cm}^{-1}$, qualitative Darstellung ohne Angabe der Konzentrationen und weiterer Messbedingungen) [Quelle Dräger Medical GmbH]

Neben der Stärke und der Spezifik der Absorptionen müssen auch die in der Messung auftretenden Konzentrationsbereiche und die geforderten Messgenauigkeiten für die einzelnen Gaskomponenten berücksichtigt werden. Gleichzeitig soll die Messung aber in einer Messzelle mit einer einheitlichen Absorptionslänge ausgeführt werden. Daraus ergibt sich die Forderung, dass die Analyse in beiden Wellenlängenbereichen erfolgen muss. 

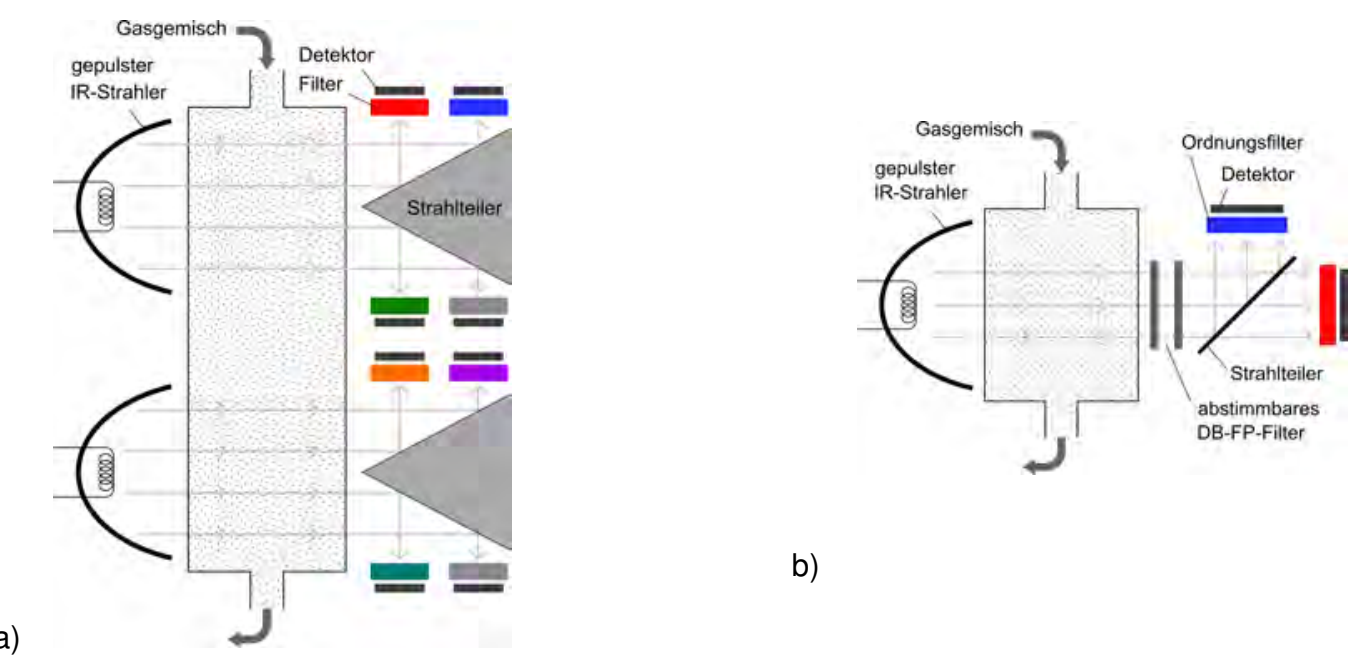

b)

Bild 10: a) Anästhesiegassensor nach dem Stand der Technik mit zwei Vierkanaldetektoren mit interner Strahlteilung, b) In Entwicklung befindlicher neurartiger Sensor mit DB-FP-Modul

Der Stand der Technik für diese Messaufgabe besteht in der Nutzung eines Filterrades oder von Mehrkanaldetektoren. Bild 10a zeigt den Aufbau eines solchen Sensors, wie er derzeit von der Dräger Medical GmbH angeboten wird. Er besteht im Prinzip aus zwei separaten Sensoren mit jeweils eigenem Strahler, Gasküvette und einem Vierkanaldetektor. Letzterer besitzt eine gemeinsame Eintrittsapertur für alle vier Kanäle und einen internen Strahlteiler. Dadurch ist er sehr unempfindlich gegenüber Driften durch Alterung oder evtl. Verunreinigungen im Strahlengang. Werden die beiden Vierkanaldetektoren nun durch das oben beschriebene DB-FP-Detektormodul ersetzt, so ist eine wesentlich kompaktere und damit auch kostengünstigere Sensorkonstruktion möglich. Wie in Bild 10b dargestellt, wird der komplette Sensor auf eine Messstrecke reduziert. Es verringert sich gleichzeitig das auszutauschende Gasvolumen und damit die Ansprechzeit des Sensors. Mit ersten Prototypen konnte im Prinzip die gleiche Leistungsfähigkeit wie in der bewährten Lösung nachgewiesen werden. Ein weiteres Ziel der Entwicklung besteht darin, die Messfrequenz deutlich zu steigern.

\subsection{Weitere Anwendungsbereiche in der Gasanalyse}

Potentielle Anwendungsbereiche sind überall dort zu finden, wo eine Mehrkomponentenmessung mit moderatem spektralem Auflösungsvermögen benötigt wird. Ein solcher Fall ist $z$. B. die Atemalkoholmessung, bei der nicht nur die Ethanolkonzentration möglichst genau zu bestimmen ist, sondern auch Querempfindlichkeiten gegen eine Vielzahl von Stoffen auszuschließen sind. Diabetiker atmen Azeton aus, Personen mit Leber- oder Stoffwechselerkrankungen möglicherweise Ammoniak, Raucher Kohlenmonoxid - um nur einige Beispiele zu nennen.

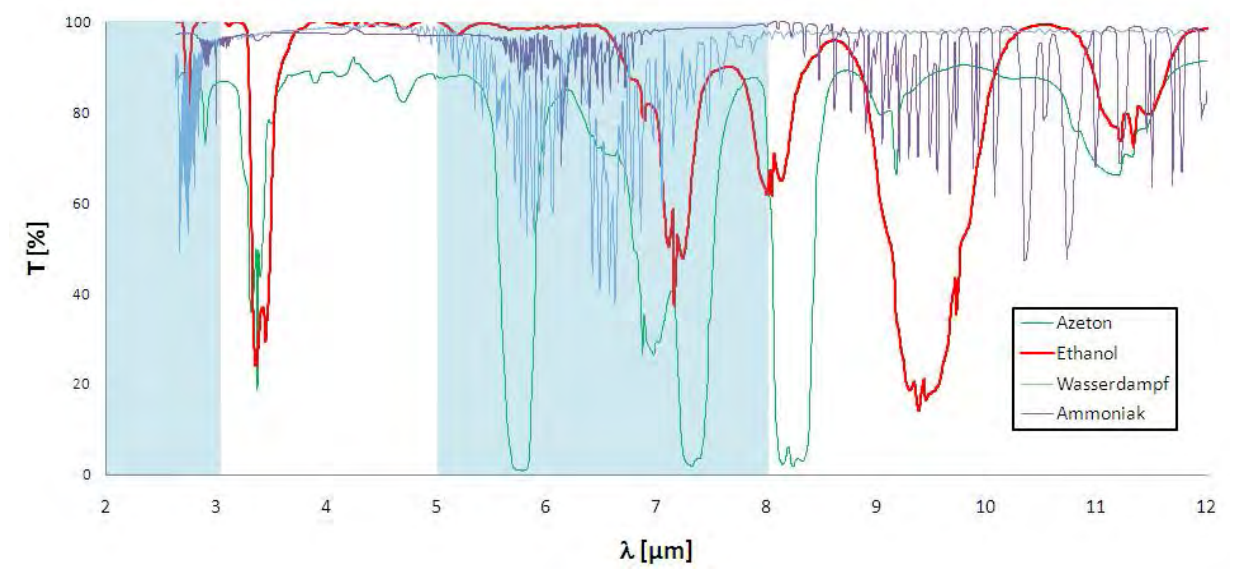

Bild 11: Auswahl von Gasspektren, die für die Atemalkoholmessung relevant sind

Um gerichtsverwertbare Messergebnisse zu garantieren, werden daher mehrere parallel arbeitende Sensoren mit unterschiedlichen Messprinzipen, z. B. IR-optische und elektrochemische Sensoren, 
benutzt. Letztere leiden aber besonders stark unter Querempfindlichkeiten und bieten zur IR-Messung daher nur einen begrenzten Zusatznutzen. Die Erweiterung des IR-Sensors zu einem spektrometrischen System verspricht hier einige Vorteile. Die in Bild 11 dargestellten Spektren verdeutlichen diesen Sachverhalt. Auch hier ist vorteilhaft, Spektren insbesondere im langwelligen Spektralbereich messen zu können.

Neben diesen ausführlicher erläuterten Anwendungsfällen gibt es zahlreiche weitere, die für den Einsatz von FP-Mikrospektrometern prädestiniert sind. Als Beispiele seinen noch die Überwachung des Gärprozesses in Biogasfermentern oder die Messung Ethlylen und Ethanol bei der Lagerung bzw. beim Reifeprozess von Früchten genannt.

\subsection{Absorptionsmessungen in flüssigen Medien}

Für die Analyse von flüssigen ist ein großer Wellenlängenbereich von 5,5 - 11 $\mu \mathrm{m}$ von Interesse, welcher durch die oben beschriebenen Entwicklungen abgedeckt werden kann. Als Messverfahren dominiert hier das ATR-Prinzip (abgeschwächte Totalreflexion). Hierbei taucht ein hochbrechender Kristall in die Flüssigkeit. An den Grenzflächen zum Medium wird die IR-Strahlung totalreflektiert, die Welle dringt aber zu einem kleinen Teil in das Medium ein (evaneszente Welle) und wird je nach Stoffzusammensetzung absorbiert. Entsprechende Spektrometer sind oft mit linearen Verlaufsfiltern mit einer Bandbreite von rund $2 \%$ der Wellenlänge ausgerüstet. Um die gewünschte Wellenlänge zu selektieren muss entweder das Filter bewegt oder mit einem Zeilendetektor kombiniert werden. Verlaufsfilter und Zeilendetektoren sind relativ teure Komponenten, die den Preis für den Sensor in die Höhe treiben. Wird stattdessen ein abstimmbares FP-Filter eingesetzt, ist auch hier eine deutlich kompaktere und preisgünstigere Konstruktion möglich.

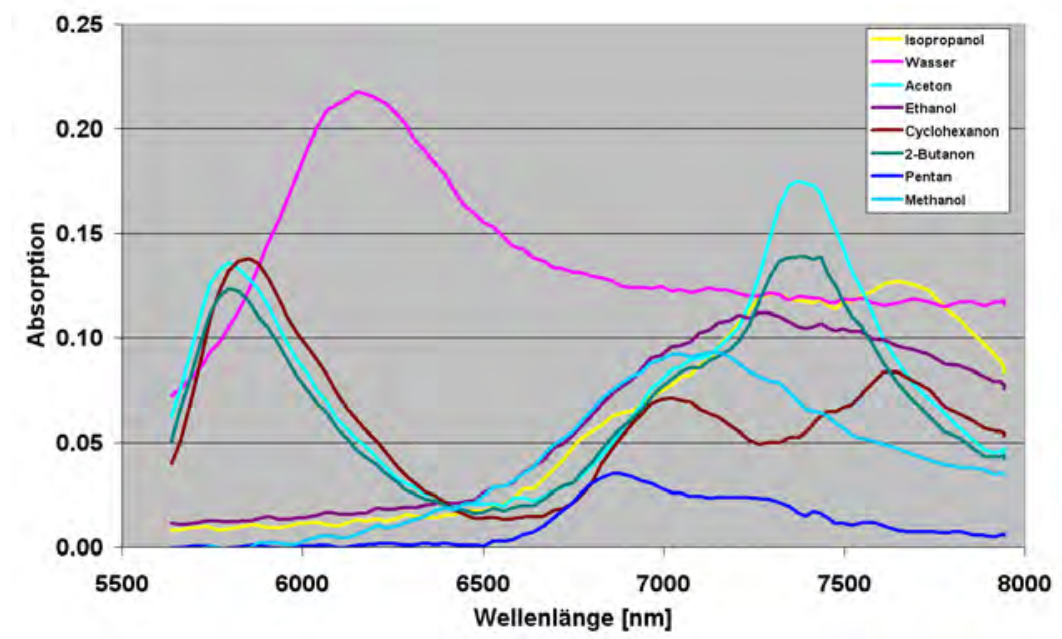

a)

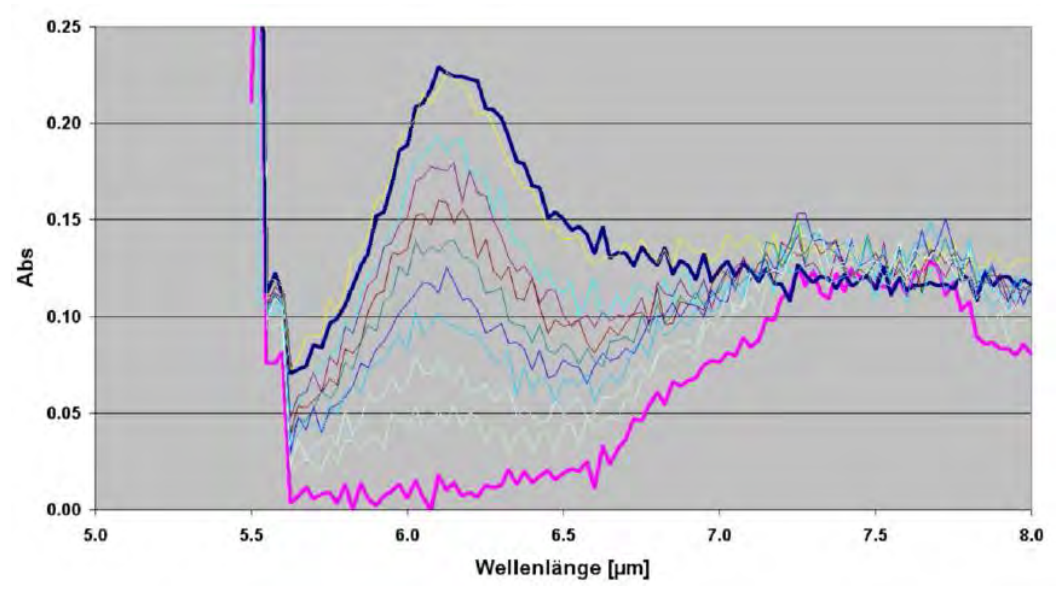

b)

c)

Bild 12: a) ATR-Spektren verschiedener Lösungsmittel gemessen mit FP-Filter im Wellenlängenbereich 5,5 - 8 $\mu \mathrm{m}$, b) Kalibrierspektren des Systems Isopropanol - Wasser aufgenommen mit FP-Filter im Wellenlängenbereich 5,5-8 $\mu \mathrm{m}, \mathrm{c}$ ) Prozessspektrometer mit ATR-Sonde von Endress+Hauser [Quelle Endress+Hauser] 
Die Firma Endress+Hauser hat FP-Filter für den Wellenlängenbereich 5,5-8 $\mu \mathrm{m}$ und $8-11 \mu \mathrm{m}$ in eigenen Prozessspektrometern (Bild 12c) für verschiedene Anwendungsfälle getestet. Einige Beispielspektren, welche im Rahmen der Untersuchungen gemessen wurden, sind in den Diagrammen in Bild 12a und $\mathrm{b}$ dargestellt.

Die Bestimmung des Zustand bzw. der Alterung von Schmierstoffen in großtechnischen Anlagen ist wichtig, um einen zuverlässigen Betrieb zu gewährleisten aber andererseits auch um unnötige Kosten durch einen zu frühen Austausch zu sparen. Dies trifft in besonderem Maße auf Offshore Windkraftanlagen zu. Der hohe Aufwand für einen Wechsel des Getriebeöls schlägt mit einigen tausend Euro zu Buche. Hier wird ein Sensor für eine kontinuierliche Online-Überwachung in der Anlage benötigt. Ein derartiger Sensor, basierend auf einer IR-ATR-Messung ist in [14] beschrieben worden. Im Rahmen dieser Entwicklung wurden auch FP-Sensoren im Wellenlängenbereich $3-5 \mu \mathrm{m}$ getestet. Schließlich wurde zwar eine Lösung mit einem Verlaufsfilter im Spektralbereich 5,5-11 $\mu \mathrm{m}$ und einem pyroelektrischen Zeilendetektor favorisiert. Das lag aber hauptsächlich daran, dass entsprechende langwellige FP-Sensoren zu diesem Zeitpunkt noch nicht verfügbar waren.

\section{Entwicklung von zeitlich und spektral höher auflösenden FP-Filtern}

Wie bereits erläutert wurde, eröffnet die Erweiterung des abstimmbaren Wellenlängenbereichs in das langwellige Infrarot neue Perspektiven für Anwendungen, die sich im mittleren Infrarotbereich von 3 $5 \mu \mathrm{m}$ nicht ohne weiteres lösen lassen. Ein Ausweichen zu höheren Wellenlängen ist aber nicht immer möglich oder praktikabel. Um mit einem FP-Spektrometer im Bereich 3-5 $\mu \mathrm{m}$ trotzdem ausreichend selektiv messen zu können, ist vor allen Dingen eine deutlich gesteigerte spektrale Auflösung nötig. Eine weitere Beschränkung besteht bisher außerdem in der relativ niedrigen Dynamik der Filter (Abstimmgeschwindigkeit) und des pyroelektrischen Detektors.

Diese Punkte sollen im Rahmen eines aktuellen Entwicklungsvorhabens gelöst oder deutlich verbessert werden. Grundidee ist es, ein mikromechanisches Fabry-Perot-Interferenzfilter ( $\mu \mathrm{FPF}$ ) im Spektralbereich von etwa $3-4 \mu \mathrm{m}$ in der dritten bzw. vierten Interferenzordnung zu betreiben, wodurch die spektrale Auflösung auf besser als $20 \mathrm{~nm}$ reduziert werden kann. In Kombination mit einem schnellen HalbleiterPhotodetektor können Spektren direkt erfasst werden, indem das $\mu$ FPF kontinuierlich durchgestimmt wird (Sweep-Modus).
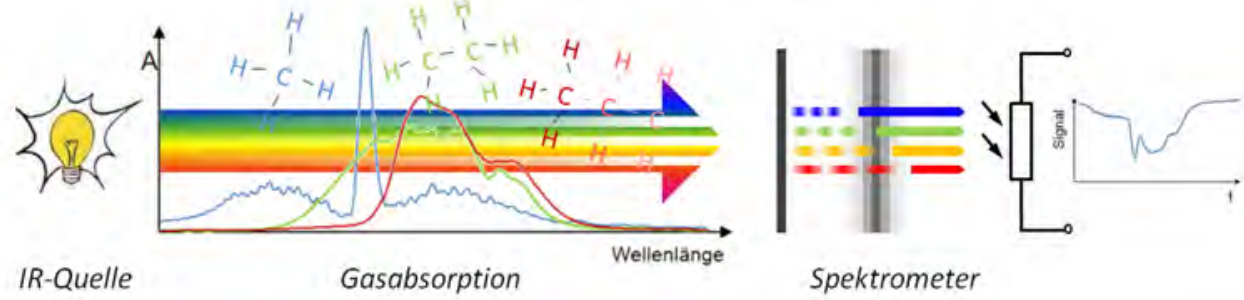

Bild 13: Grundanordnung eines Gasanalysators mit neuartigem hochauflösenden Spektralsensor

In dem Vorhaben soll eine neue Klasse von infrarotspektrometrischen Sensoren und Messverfahren entwickelt werden, welche in Geräten für die hochgenaue und schnelle Analyse von Gasgemischen, insbesondere von Kohlenwasserstoffen und weiteren Verbindungen, eingesetzt werden kann. Das Prinzip und die wesentlichen Innovationen des Vorhabens sind in Bild 13 dargestellt. Anwendungsgebiete werden in erster Linie in der Analyse von Kohlenwasserstoffgemischen, z. B. in der Messung des Energiegehalts von Erdgas oder Biogas, der Steuerung von Verbrennungsprozessen oder der Messung von flüchtigen Kohlenwasserstoffen (VOC), gesehen.

Zur Steigerung der spektralen Auflösung wird der Ansatz verfolgt, das FP-Filter in höheren Interferenzordnungen (vorzugsweise dritte oder vierte) zu betreiben. Weitere Verbesserungen werden von einer Optimierung der Technologie (Reduzierung der Defektfinesse) erwartet. Eine entsprechende Vorstudie lässt es realistisch erscheinen, dass eine Filterbandbreite von $20 . .25 \mathrm{~nm}$ erreicht werden kann. Dies stellt eine Verbesserung des Auflösungsvermögens um den Faktor 3...4 gegenüber dem Stand der Technik mit FP-Filtern in erster Ordnung (siehe Bild 14a) dar.

Nachteilig ist, dass sich mit dem FSR der Abstimmbereich in einer höheren Interferenzordnung reduziert, im Falle des hier gezeigten Beispiels auf $3,1-3,7 \mu \mathrm{m}$. Für viele der avisierten Anwendungsfälle ist dies jedoch auch ausreichend. Der Auflösungsgewinn gegenüber einem Filter erster Ordnung mit ca. $75 \mathrm{~nm}$ Bandbreite ist durch die Simulation einer Messung eines Gasgemischs aus Kohlenwasserstoffen in Bild 14b illustriert. 

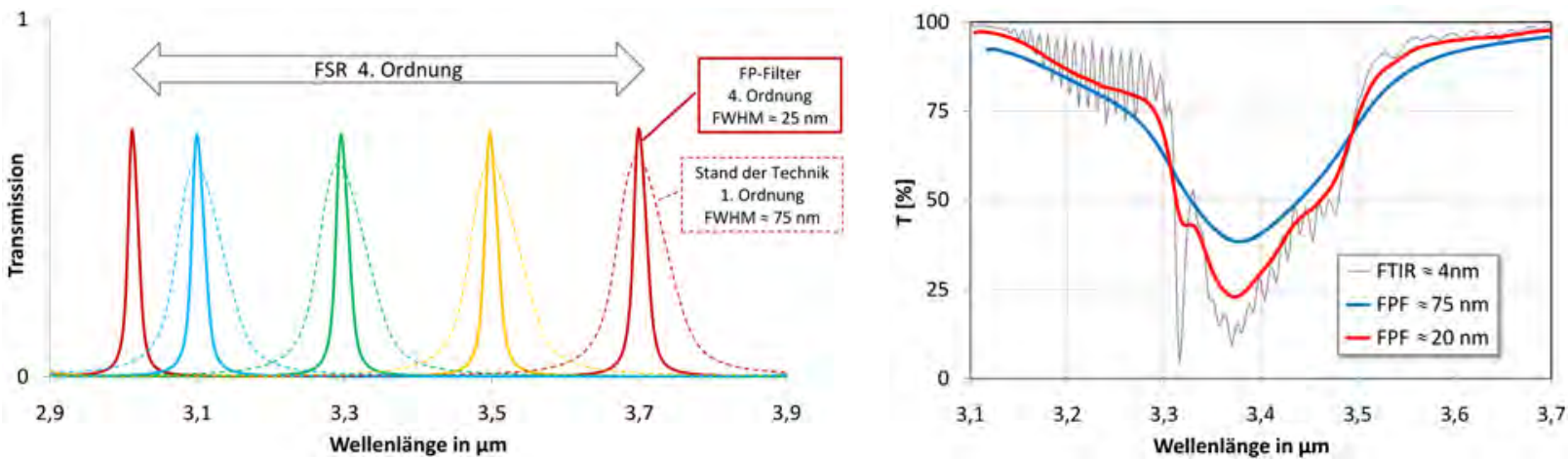

Bild 14: Simulierte Spektren für FPI in erster und vierter Ordnung, b) Auflösungsgewinn bei der Messung eines Gasgemischs (verschiedene Alkane) mit einem FP-Spektrometer durch Nutzung einer höheren Interferenzordnung und Vergleich mit einer FTIR-Referenzmessung

Eine Erweiterung auf angrenzende Spektralbereiche, um zukünftig z.B. auch die $\mathrm{CO}_{2}$-Bande (um $4,25 \mu \mathrm{m})$ zu erfassen, ist durch die Nutzung der benachbarten Interferenzordnung ebenfalls möglich. Der Aufbau eines solchen Sensors kann zu großen Teilen vom oben beschriebenen Dual-Band-Sensor adaptiert werden. Simulierte Spektren für ein entsprechendes Filter sind in Bild 15 dargestellt. Erste Ergebnisse aus diesem Projekt sind innerhalb der nächsten zwei Jahre zu erwarten.

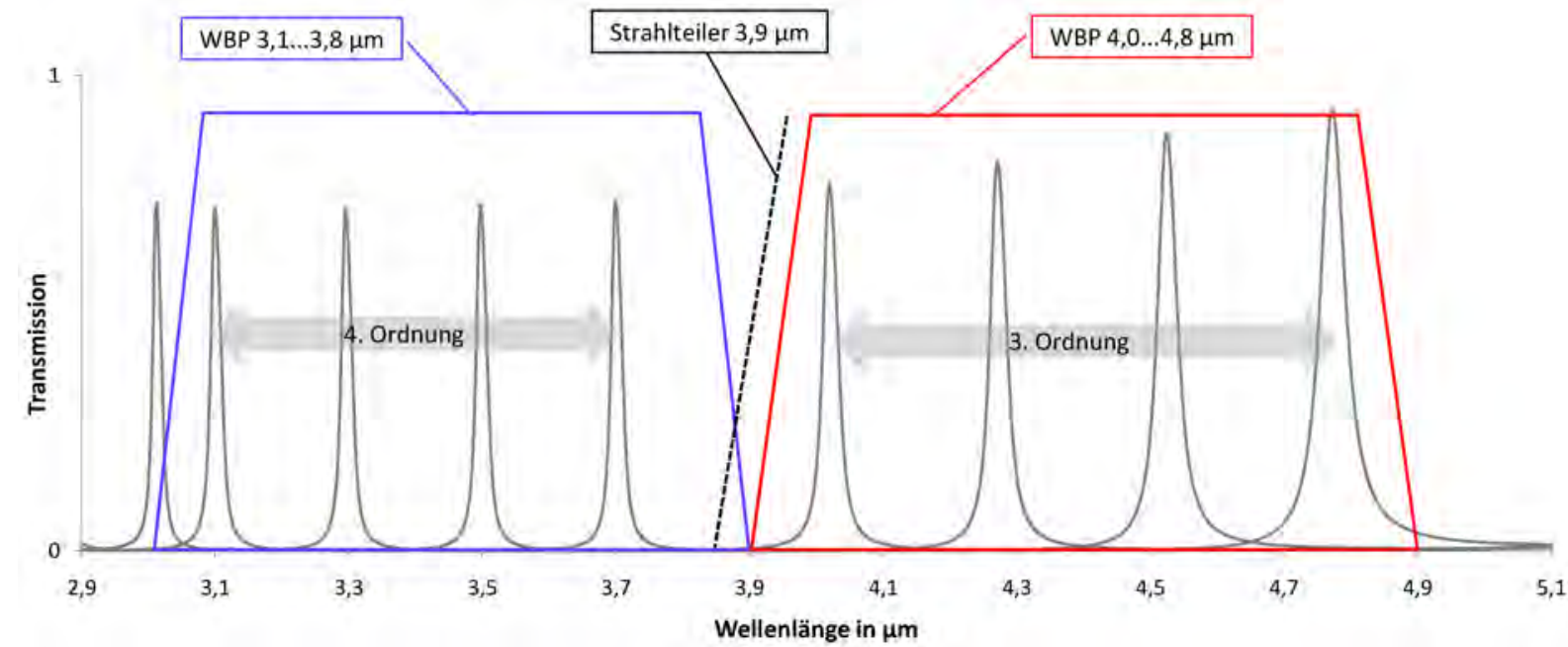

Bild 15: Funktionsprinzip eine hochauflösenden Dual-Band-Sensors für Kohlenwasserstoffe und $\mathrm{CO}_{2}$ durch simultane Nutzung zweier benachbarter Interferenzordnungen

\section{Zusammenfassung}

Neben den bereits kommerziell erhältlichen FP-Sensoren für den Wellenlängenbereich $3-5 \mu \mathrm{m}$ ist eine Erweiterung der Technologie hin zu höheren Wellenlängenbereichen und zu mehr Stabilität gegenüber Beschleunigungskräften gelungen. Die Bandbreite der Filter liegt in allen verfügbaren Spektralbereichen im Bereich von 1,5...2,5 \% der Filterwellenlänge, was sie für viele Anwendungsbereiche der IRSpektroskopie empfiehlt. Das gilt vor allem dort, wo neben einem moderaten spektralen Auflösungsvermögen ein hoher optischer Durchsatz gefordert wird. Dies wurde an einigen Beispielapplikation der medizinischen Gasanalyse und der industriellen Prozessmesstechnik erläutert. Neben der kontinuierlichen Verbesserung der Technologie ist in einem aktuellen Vorhaben die Entwicklung von höher auflösenden FP-Filtern geplant. 


\section{Literatur}

[1] J. Staab, Industrielle Gasanalyse. Oldenbourg, Verlag,München, Wien, 1994

[2] H. Günzler, H.-U. Gremlich, IR-Spektroskopie, Wiley-VCH, Weinheim, 2003

[3] D. Rossberg, "Silicon micromachined infrared sensor with tunable wavelength selectivity for application in infrared spectroscopy" Sens. Actuators, A 47, 413-416, (1995)

[4] A. Lehto, M. Orpana, M. Blomberg, "Electrically tunable fabry-perot interferometer produced by surface micromechanical techniques for use in optical material analysis" European patent application EP0668490, (1995)

[5] J. Antoszewski, A. Keating, K. Winchester, T. Nguyen, D. Silva, C. Musca, J. Dell, O. Samardzic, L. Faraone, "Tunable Fabry-Perot filters operating in the 3 to $5 \mu \mathrm{m}$ range for infrared micro-spectrometer applications" Proc. SPIE, Vol. 6186, 618608 (2006)

[6] M. Noro, K. Suzuki, N. Kishi, H. Hara, T. Watanabe, and H. Iwaoko, "CO2/H2O gas sensor using tunable Fabry-Perot filter with wide wavelength range", Proc. IEEE MEMS 2003 Conf., 319-322 (2003)

[7] J.M. Vaughan, The Fabry-Perot Interferometer, IOP Publishing Ltd.

[8] E. D. Palik, H. Boukari, R. W, Gammon, "Experimental study of the effect of surface defects on the finesse and contrast of a Fabry-Perot interferometer,", Appl. Opt. 35, 38-50 (1996)

[9] J. Moon, A. M. Shkel, "Performance limits of a micromachined tunable-cavity filter", Proc. Int. Conf. MSM 2001, pp. 278-281 (2001)

[10] N. Neumann, M. Ebermann, S. Kurth, K. Hiller, "Tunable infrared detector with integrated micromachined Fabry-Perot filter.", J.Micro/Nanolith. MEMS MOEMS 7(2), 021004-01 - 021004-9 (2008)

[11] M. Ebermann, N. Neumann, K. Hiller, E. Gittler, S. Kurth, "Recent Advances in Expanding the Spectral Range of MEMS Fabry-Pérot Filters", Proc. SPIE, Vol. 7594, (2010)

[12] M. Meinig, S. Kurth, K. Hiller, M. Ebermann, E Gittler, T. Geßner, "Entwurf und Technologie eines Dualband Fabry-Pérot-Filters für den mittleren Infrarotbereich mit zwei beweglichen Reflektoren", 10. Fachtagung Mikrosystemtechnik, Chemnitz, 2010

[13] N. Neumann, M. Ebermann, E. Gittler, M. Meinig, M.; S. Kurth; K. Hiller, "Uncooled IR Sensors with Tunable MEMS Fabry-Pérot Filters for the Long-Wave Infrared Range“, Proc. IEEE Sensors 2010 Conf., 2383-2387 (2010)

[14] B. R. Wiesent, D. D. Dorigo,A. W. Koch: "Suitability of tunable Fabry-Perot spectrometers for condition monitoring purposes of gear oils in offshore wind turbines", Proceedings IRS ${ }^{2}$ Sensor+Test Konferenz, 2010 